\title{
Comparison between two- and three-dimensional scoring of zebrafish response to psychoactive drugs: when is three- dimensional analysis needed?
}

\author{
Simone Macrì ${ }^{1,2}$, Romain JG Clément ${ }^{1}$, Chiara Spinello ${ }^{1}$, Maurizio Porfiri ${ }^{\text {Corresp. } 1,3}$ \\ 1 Department of Mechanical and Aerospace Engineering, New York University, Brooklyn, New York, United States \\ 2 Centre for Behavioural Sciences and Mental Health, Istituto Superiore di Sanità, Rome, Italy \\ 3 Department of Biomedical Engineering, New York University, Brooklyn, New York, United States \\ Corresponding Author: Maurizio Porfiri \\ Email address: mporfiri@nyu.edu
}

Zebrafish (Danio rerio) have recently emerged as a valuable laboratory species in the field of behavioral pharmacology, where they afford rapid and precise high-throughput drug screening. Although the behavioral repertoire of this species manifests along three dimensions (3D), most of the efforts in behavioral pharmacology rely on 2D projections acquired from a single overhead or front camera. We recently showed that, compared to a 3D scoring approach, 2D analyses could lead to inaccurate claims regarding individual and social behavior of drug-free experimental subjects. Here, we examined whether this conclusion extended to the field of behavioral pharmacology by phenotyping adult zebrafish, acutely exposed to citalopram (30 mg/L, $50 \mathrm{mg} / \mathrm{L}$, and $100 \mathrm{mg} / \mathrm{L}$ ) or ethanol $(0.25 \%, 0.50 \%$, and $1.00 \%)$, in the novel tank diving test over a six-minute experimental session. We observed that both compounds modulated the time course of general locomotion and anxiety-related profiles, the latter being represented by specific behaviors (erratic movements and freezing) and avoidance of anxiety-eliciting areas of the test tank (top half and distance from the side walls). We observed that 2D projections of 3D trajectories (ground truth data) may introduce a source of unwanted variation in zebrafish behavioral phenotyping. Predictably, both 2D views underestimate absolute levels of general locomotion. Additionally, while data obtained from a camera positioned on top of the experimental tank are similar to those obtained from a 3D reconstruction, 2D front view data yield false negative findings. 


\section{Comparison between two- and three-dimensional scoring of zebrafish}

2 response to psychoactive drugs: when is three-dimensional analysis needed?

3

4 5

6

Simone Macrì ${ }^{1,2}$, Romain J. G. Clément ${ }^{1}$, Chiara Spinello ${ }^{1}$, Maurizio Porfiri1 ${ }^{1,3}{ }^{*}$ 5

${ }^{1}$ Department of Mechanical and Aerospace Engineering, New York University, Tandon School of Engineering, 6 MetroTech Center, Brooklyn, NY 11201, USA

${ }^{2}$ Centre for Behavioural Sciences and Mental Health, Istituto Superiore di Sanità, Viale Regina Elena 299, 00161, Rome, Italy

${ }^{3}$ Department of Biomedical Engineering, New York University, Tandon School of Engineering, 6 MetroTech Center, Brooklyn, NY 11201, USA

${ }^{*}$ Corresponding Author:

Maurizio Porfiri

6 MetroTech Center, Brooklyn, NY, 11201, USA

Email address: mporfiri@nyu.edu

\section{Abstract}

Zebrafish (Danio rerio) have recently emerged as a valuable laboratory species in the field of behavioral pharmacology, where they afford rapid and precise high-throughput drug screening. Although the behavioral repertoire of this species manifests along three dimensions (3D), most of the efforts in behavioral pharmacology rely on 2D projections acquired from a single overhead or front camera. We recently showed that, compared to a 3D scoring approach, 2D analyses could lead to inaccurate claims regarding individual and social behavior of drug-free experimental subjects. Here, we examined whether this conclusion extended to the field of behavioral pharmacology by phenotyping adult zebrafish, acutely exposed to citalopram (30 $\mathrm{mg} / \mathrm{L}, 50 \mathrm{mg} / \mathrm{L}$, and $100 \mathrm{mg} / \mathrm{L})$ or ethanol $(0.25 \%, 0.50 \%$, and $1.00 \%)$, in the novel tank diving test over a six-minute experimental session. We observed that both compounds modulated the time course of general locomotion and anxiety-related profiles, the latter being represented by specific behaviors (erratic movements and freezing) and avoidance of anxiety-eliciting areas of 
31 the test tank (top half and distance from the side walls). We observed that 2D projections of 3D

32 trajectories (ground truth data) may introduce a source of unwanted variation in zebrafish

33

34

35 behavioral phenotyping. Predictably, both 2D views underestimate absolute levels of general locomotion. Additionally, while data obtained from a camera positioned on top of the experimental tank are similar to those obtained from a 3D reconstruction, 2D front view data yield false negative findings.

Keywords: anxiety; automated tracking; citalopram; ethanol; novel tank diving test.

\section{Introduction}

Preclinical animal models constitute a central tool to detail the fundamental mechanisms underlying the expression of human emotions in physiological and pathological conditions (Haller \& Alicki 2012). Within this framework, several experimental models have been proposed to investigate the neurobiological processes underlying anxiety (Davis et al. 2010; Hart et al. 2010), an evolutionarily preserved adaptive emotion, normally occurring as an anticipatory response to a potential threat (Bateson et al. 2011). The adaptive value of anxiety resides in the fact that it limits the negative outcomes associated with a potential threat (Nesse 1999). Notwithstanding its adaptive nature, inappropriate (context-independent) or excess anxiety may often culminate in anxiety-related disorders that require medical attention (Bateson et al. 2011).

In parallel with the aforementioned evolutionary roots, the underlying biological determinants of anxiety are very well conserved across different taxa. For example, the neuroendocrine machinery activated in response to external stressors exhibits striking homologies and analogies among fish (Bernier \& Peter 2001), birds (Lynn \& Kern 2018), rodents (Macrì \& Wurbel 2006), monkeys (Parker et al. 2012), and humans (Rodrigues et al. 2009). Likewise, neurotransmitters such as serotonin have been associated with anxiety-related behaviors in species as diverse as fish (Fossat et al. 2014), birds (Hogg et al. 1994), humans (Caspi et al. 2003), and sheep (Lee et al. 2016).

Although rodents have traditionally constituted the species of choice in this field (Hart et al. 2010; Kalueff et al. 2007), zebrafish have recently emerged as an extremely promising experimental species (Fontana et al. 2018; Shams et al. 2018; Stewart et al. 2014). The success of 
61 this freshwater species rests upon several advantages that range from genetic and neuroanatomic

62 similarities between zebrafish and humans (Howe et al. 2013), to their small size and high

63

64 reproductive rates favoring the execution of high-throughput studies (Kalueff et al. 2014). In addition, the possibility to dissolve substances in water allows for the non-invasive administration of drugs readily absorbed through the gills (Tran \& Gerlai 2013). These characteristics designate zebrafish as a fundamental tool in the field of psychopharmacology whereby they allow the preliminary screening of numerous drugs within spaces and time frames much smaller than those required by laboratory mammals (McCarroll et al. 2016).

High-throughput behavioral experiments on zebrafish generally share the following methodological structure: administration of experimental treatments (e.g., administration of water-soluble compounds and exposure to fear-evoking or social stimuli), videorecording of observable phenotypes, offline scoring of video, coding of the observed behaviors, and data analysis (Stewart et al. 2014). Traditional behavioral phenotyping leveraged the use of a single camera positioned on top or in front of the experimental tank and the subsequent use of behavioral scoring software, in which the phenotype of interest had to be input by a trained observer (Cianca et al. 2013; Spinello et al. 2013). Albeit extremely productive, this approach was prone to observer bias and has been recently complemented by tracking algorithms capable of automatically coding and scoring zebrafish behavior with limited human supervision (Delcourt et al. 2018; Franco-Restrepo et al. 2019; Nema et al. 2016; Perez-Escudero et al. 2014).

However, from the two-dimensional (2D) view offered by a single video-camera it is impossible to phenotype the 3D swimming pattern exhibited by zebrafish. This consideration prompted the design and development of experimental platforms capable of investigating zebrafish behavior adopting a 3D approach (Cachat et al. 2011; Maaswinkel et al. 2013; Macrì et al. 2017; Stewart et al. 2015). We recently demonstrated that the limitation of 2D scoring methods extends beyond the geometrical underestimation of swimming paths (3D trajectories being longer than their 2D projections by definition), and may result in numerous false positive and false negative findings (Macrì et al. 2017). Specifically, we first tested zebrafish in conventional binary choice behavioral assays, and then analyzed group differences based on 3D or 2D (top and front views) trajectories. This analysis demonstrated that 2D views generated approximately $20 \%$ of false findings, being represented by inappropriate reporting of significant 
92 inter-group differences in spite of undistinguishable ground truth data (false positives) or failure

93 to detect significant results in instances in which experimental groups belonged to different

94

95

96

97

98 populations (false negatives) (Macrì et al. 2017).

In the present study, we aimed at prospectively investigating whether 3D scoring of zebrafish behavior may also benefit zebrafish research in the field of behavioral response to psychoactive substances. To this aim, we exposed experimentally naïve zebrafish to drugs capable of modulating anxiety-related behaviors in both humans and zebrafish (Cianca et al. 2013; Sackerman et al. 2010), and then analyzed their phenotype in response to an anxietyprovoking test paradigm in 3D or in 2D (top and front views). Specifically, we investigated the behavior of zebrafish in a novel tank diving test in response to the administration of the selective serotonin reuptake inhibitor citalopram $(30 \mathrm{mg} / \mathrm{L}, 50 \mathrm{mg} / \mathrm{L}$, and $100 \mathrm{mg} / \mathrm{L})$ or ethanol $(0.25 \%$, $0.50 \%$, and $1.00 \%$ ). These substances were selected based on their known efficacy in modulating anxiety-related behavior, their water-soluble nature, and their non-restricted use. These factors contribute to the replicability of the study and reproducibility of the results, while shedding light on the effects of widely used and abused substances, see, e.g., (Pannia et al. 2014; Sackerman et al. 2010). The novel tank diving test has already been validated as a locomotion- and anxietyrelated behavioral test (Cachat et al. 2010). Therein, anxiety is measured through the evaluation of fish position in the water column, swimming speed, erratic movements, and freezing, as functions of the time spent in the experimental tank from the initial release.

The aim of this study was twofold: first, we sought to replicate existing findings indicating that ethanol (Pannia et al. 2014) and citalopram (Sackerman et al. 2010) modulate anxiety in zebrafish (predictive validity of the assay), and then we aimed at testing whether the experimental advantages afforded by 3D scoring in drug-free states (Macrì et al. 2017) also extend to zebrafish psychopharmacology. Grounded in our previous work, we anticipated 2D views to be characterized by reduced absolute locomotion values compared to 3D trajectories. Most importantly, in the light of the high rate of false findings observed in drug-free conditions (Macrì et al. 2017), we expected the predictive validity of 2D trajectories to be potentially jeopardized. This hypothesis rests on the fact that, when exposed to psychoactive substances, fish may exhibit a series of responses that vary in space and time. For example, increased anxiety may reflect in a progressive reduction in general locomotion, increased freezing, erratic movements, and preference for the bottom of the experimental tank. These patterns manifest 
123 differentially depending on the time spent in the experimental apparatus (with preference for the

124 bottom varying with the prolonged exposure), and on the view (i.e., top or front view). For

125 example, while horizontal erratic movements are best detected through a top view, geotaxis can

126 be appropriately scored only from a side view. Therefore, we hypothesized that the specific view

127 may reflect into a bias in detecting time-dependent effects of psychoactive drugs, thereby

128 potentially generating view $\times$ drug $\times$ experimental-progression effects.

\section{Materials and methods}

131

132

133

134

135

136

137

138

139

140

141

142

143

144

145

146

147

148

149

150

151

152

\subsection{Animal care and maintenance}

The experiments and analysis were performed and reported according to the ARRIVE guidelines (Kilkenny et al. 2010). A total of 112 wild-type adult zebrafish (Danio rerio), with a 1:1 male/female ratio were used in this study. The fish were purchased from Carolina Biological Supply Co. (Burlington, NC, USA), and housed in 10 L (2.6 gallons) vivarium tanks (Pentair Aquatic Eco-Systems Locations, Cary, NC, USA), with a density of no more than 10 fish per tank. Fish were kept under a $12 \mathrm{~h}$ light/12 h dark photoperiod (Cahill 1996), and fed with commercial flake food (Hagen Corp. Nutrafin max, Mansfield, MA, USA) once a day, approximately at 7 PM. Water parameters of the holding tanks were regularly checked, and temperature and $\mathrm{pH}$ were maintained at $26^{\circ} \mathrm{C}$ and $7.2 \mathrm{pH}$, respectively. Regular tap water was used with the addition of a stress coat (AquaSafe plus, Tetra, Spectrum Brands Inc., Germany) to remove chlorine and chloramines. Prior to the beginning of the experiments, fish were acclimatized in the holding facility for a period of 12-15 days.

The number of fish used in the study - compatible with obtaining sufficiently reliable and biologically relevant data - was estimated through a power analysis. Briefly, we computed the minimum required sample size considering the two-tailed Student $t$ test for independent groups using the following values, based on the results of previous studies (Abaid et al. 2012; Cianca et al. 2013; Spinello et al. 2013): (i) standard deviation homogeneous among groups s = 0.23; (ii) Type I error probability $\mathrm{a}=0.05$ and power $1-\mathrm{b}=0.80$ (conventional values); and (iii) minimum difference between control and treatment group means $\mathrm{D}=0.17$. The sample size resulting from this calculation was 15 subjects per group. To promote the generalizability of our findings, we conducted experiments on both males and females. We thus increased the sample size to 16 per 
153 group (eight males and eight females). We estimated that a sample size of 16 subjects (per

154 group) would have $80 \%$ power to detect a 0.60 effect size on the principal outcome measures

155 with a two-tailed significance level of 0.05.

156

157

\subsection{Experimental setup}

158 To obtain 3D trajectories, we used two Flea 3 USB high resolution cameras (Point Grey

159 Research Inc., Richmond, British Columbia, Canada), one overhead and one in front. Videos

160 were acquired at 30 frames per second, using a resolution of $588 \times 264$ pixels from the top view

161 and $652 \times 360$ pixels from the front view; the typical size of one video from the top view was 6-7

162 gigabytes, while the front view was 9-10 gigabytes. The dimensions of the test tank were $29 \mathrm{~cm}$

163 (length) $\times 14 \mathrm{~cm}$ (height) $\times 8.5 \mathrm{~cm}$ (width) and water $13 \mathrm{~cm}$ deep, similar to tanks used in

164 comparable studies (Egan et al. 2009). The distance from the overhead camera and the bottom of

165 the tank was $62 \mathrm{~cm}$; while the distance from the front camera and the back of the tank was 60

$166 \mathrm{~cm}$

To maximize the visual contrast and ease automatic tracking, the bottom of the tank was

168

169

170

171

172

173

174

175

176

177

178

179

180

181

182

183

lined with white contact paper. The two short sides of the tank were covered with black contact paper to prevent reflection. On the other hand, the two long sides were kept transparent to allow data acquisition and avoid position bias (i.e., a potential side preference had one side been kept transparent for data acquisition and the other kept opaque). The experimental arena was surrounded by black curtains to prevent light reflection and visual disturbance from the outside.

\subsection{Experimental procedure}

Experiments, performed in June 2018, were conducted on seven groups, each consisting of 16 subjects (eight males and eight females). Specifically, the experimental design entailed one control group exposed to vehicle (water), three groups treated with citalopram $(30 \mathrm{mg} / \mathrm{L}, 50$ $\mathrm{mg} / \mathrm{L}, 100 \mathrm{mg} / \mathrm{L})$, and three groups treated with ethanol $(0.25 \%, 0.50 \%, 1.00 \%$ ethanol/water solution in volume/volume \%). The fish were randomly allocated to each of the seven conditions in the following way. The conditions were randomly distributed over several weeks, testing eight subjects per day (four in the morning and four in the afternoon). We balanced sex across conditions, and conditions across mornings and afternoons. Male and female fish were kept in separate tanks; in total, fish were housed in 12 tanks. At the beginning of each test session, we 
184 sampled one subject from a tank. Such a tank was different from that out of which we chose the 185 previous subject tested in the same condition. This procedure guaranteed that potential tank 186 effects were distributed evenly across all experimental groups.

187

188 189 190

Due to technical issues, four trials had to be discarded: this resulted in a slight reduction in the number of subjects in the $100 \mathrm{mg} / \mathrm{L}$ citalopram group (15 subjects instead of 16) and in both the $0.25 \%$ and $1.00 \%$ ethanol groups (14 and 15 subjects instead of 16 , respectively). Following (Sackerman et al. 2010), we measured the effect of acute exposure to citalopram by treating the fish to the substance for five minutes before testing it. Following previous work on the effect of ethanol by our group (Cianca et al. 2013), we measured the effect of exposure to ethanol over a one-hour period. In the interest of reducing the number of subjects used in animal experimentation, the same control subjects were used to test the effects of citalopram and ethanol. Fish were treated and tested in isolation.

Since these substances required a differential pre-exposure time (five minutes for citalopram and one hour for ethanol), we devised a common procedure for vehicle, citalopram and ethanol. Thus, one hour before testing, fish were placed in a $500 \mathrm{~mL}$ beaker filled with 450 $\mathrm{mL}$ of the following fluid: water for control and citalopram groups, or a solution of ethanol $(0.25 \%, 0.50 \%$, and $1.00 \%)$ for the other groups. Five minutes before testing, an additional 50 $\mathrm{mL}$ of fluid were slowly added to the beaker over a period of 20-30 seconds. These $50 \mathrm{~mL}$ were constituted by either water (the control group), an ethanol solution of the concentration already present in the beaker (the ethanol groups), or a concentrated solution of citalopram that resulted in a final concentration in the beaker of $30 \mathrm{mg} / \mathrm{L}, 50 \mathrm{mg} / \mathrm{L}$, or $100 \mathrm{mg} / \mathrm{L}$ (the citalopram condition). Fish were left in the beaker for five minutes, at the end of which they were transferred to the test tank and recorded for six minutes.

Simultaneous recording from both cameras was initiated before transferring the fish into the test tank. In addition, at the beginning of the recording, a laser beam, visible from both cameras, was pointed into the test tank in order to ensure later synchronization of both video streams. At the end of the experiment, the fish was hand-netted into a separate tank. All the experiments were performed at the New York University Tandon School of Engineering in Brooklyn NY (USA) in accordance with relevant guidelines and regulations, with National Institutes of Health guide for the care and use of Laboratory animals (NIH Publications 
214 No. 8023, revised 1978), and was approved by the University Animal Welfare Committee

215 (UAWC) of New York University under protocol number 13-1424.

216

217

\subsection{Tracking and $3 D$ reconstruction}

218 Images recorded from the high-resolution cameras were processed through an in-house

219

220

221

222

223

224

225

226

227

228

229

230

231

232

233

234

235

236

237

238

239

240

241

242

243 developed tracking software, see (Butail et al. 2013) for a detailed description. (The software is available for download at https://github.com/sach1tb/peregrine.) The top and front view cameras provided time series of the trajectory projected onto the $x-y$ and $x-z$ planes, respectively. Each pair of tracks were automatically synchronized using the common $\mathrm{x}$ coordinate along length of the tank. The time-series for each $\mathrm{x}$ coordinate of the pair were shifted relative to each other and the relative shift producing the smallest difference was selected. Once synchronized, the tracks from the top view and from the front views were combined to construct the $\mathrm{x}, \mathrm{y}$, and $\mathrm{z}$ coordinates of the trajectory in the three-dimensional space (see Fig. 1 for a representative trajectory exhibited by a control subject).

Reconstructed trajectories were used to quantify the following ethogram: time spent freezing (percentage of time that the fish moved less than $2 \mathrm{~cm}$ anywhere in the tank over a rolling period of $2 \mathrm{~s}$ ), time spent wall following (percentage of time that the fish spent within 3 $\mathrm{cm}$ of any side wall or the bottom of the tank), average speed (time-average of the first-order numerical differentiation of the position time series), average peak speed (time-average of the speed values greater than the $90^{\text {th }}$ percentile), average acceleration (time-average of the magnitude of the first order numerical differentiation of the velocity time series), average peak acceleration (time-average of the acceleration values greater than the $90^{\text {th }}$ percentile), average angular speed (time-computed on the basis of a finite difference approximation of the curvature of fish trajectories), average peak angular speed (time-average of the angular velocity values greater than the $90^{\text {th }}$ percentile), and time spent in the top half of the water column. These nine measures were selected from the technical literature on zebrafish behavior in novel tank tests (Cachat et al. 2010) and their objective scoring from 3D trajectories follows our previous work (Macrì et al. 2017; Mwaffo et al. 2015).

\subsection{Statistical analyses}


244 Experiments with ethanol and citalopram were analyzed separately, but both were compared to

245 the same control condition.

246

247

248

249

250

251

252

253

254

255

256

257

258

259

260

261

262

263

264

265

266

267

268

269

270

271

272

273

274

\subsubsection{Principal component analysis on $3 D$ data}

In order to detail the specific information that can be potentially inferred from these measurements, we preliminarily conducted a principal component analysis (PCA) on nine behavioral measures, objectively scored from 3D trajectories (i.e., average speed, average peak speed, average angular speed, average peak angular speed, average acceleration, average peak acceleration, time spent freezing, time spent in the top half of the tank, and time spent in the vicinity of the walls). The PCA was aimed at detecting potential correlations among the variables and identifying underlying orthogonal factors associated with independent domains.

Only principal components with eigenvalues larger than one were retained in the analysis. For each compound (citalopram or ethanol), the loadings were varimax-rotated, and the resulting scores for each principal component were used as dependent variables in a four (citalopram: vehicle, $30 \mathrm{mg} / \mathrm{L}, 50 \mathrm{mg} / \mathrm{L}$, and $100 \mathrm{mg} / \mathrm{L}$, or ethanol: vehicle, $0.25 \%, 0.50 \%$, and $1.00 \%) \times$ six (time bins, one minute each) $\times$ two $($ sex: male, female) repeated measures analysis of variance (ANOVA) for split-plot designs. Testing males and females served the aim to access a heterogeneous experimental population and therefore improve the generalizability of our findings.

For citalopram and ethanol treatments, three principal components with eigenvalue larger than one were extracted by the PCA (Table 1), accounting for $87 \%$ of the total variance. The first principal component, accounting for $47 \%$ of the variance, reflected locomotion, with positive loadings for average speed, average peak speed, average acceleration, and average peak acceleration, and a modest negative loading for the time spent freezing. The second principal component, accounting for $26 \%$ of the variance, reflected anxiety-related behavioral patterns (behavioral anxiety) with positive loadings for average angular speed, average peak angular speed, and the time spent freezing. The third principal component, accounting for $11 \%$ of variance, reflected anxiety-related spatial preference (positional anxiety), with positive loadings for the time spent wall following, and negative loadings for the time spent in top half.

These three principal components, derived from 3D observations, were used to test the efficacy of ethanol and citalopram in modifying zebrafish behavior. It was not possible to use 
275 PCA to compare the different views since the number of variables that construct the principal

276 components in 3D was greater than that in $2 \mathrm{D}$.

277

278

\subsubsection{Statistical model to compare $2 D$ and $3 D$ analyses}

279 To investigate whether 2D projections of 3D trajectories may introduce a bias in the predictive

280

281

282

283

284

285

286

287

288

289

290

291

292

293

294

295

296

297

298

299

300

301

302

303

304

305

validity of behavioral data on each of the nine measures, we conducted another repeated measures ANOVA for split-plot designs. In this analysis, the two general models for citalopram and ethanol were, respectively: three (view: 3D, 2D top, 2D front) $\times$ four (treatment: vehicle, 30 $\mathrm{mg} / \mathrm{L}, 50 \mathrm{mg} / \mathrm{L}, 100 \mathrm{mg} / \mathrm{L}) \times \operatorname{six}($ time bins, one minute each) $\times$ two (sex: male, female), and three (view: $3 \mathrm{D}, 2 \mathrm{D}$ top, 2D front) $\times$ four (treatment: vehicle, $0.25 \%, 0.50 \%, 1.00 \%$ ethanol/water solution $) \times$ six $($ time bins, one minute each $) \times$ two $($ sex: male, female $)$ repeated measures ANOVAs. Similar to the PCA, predictions of the effect of sex were not considered.

For all ANOVAs, the distribution of the model residuals was visually inspected to verify that they were close to normality (Quinn \& Keough 2002). Statistical analyses were performed using R 3.5.0, with the aov function for ANOVAs, the prcomp function for the PCA, and the emmeans 1.3.0 package for post-hoc comparisons using the Dunnett's multiple comparisons test, comparing control to other conditions and first minute to other minutes.

While main effects of the view factor allowed assessing whether absolute values differed depending on the tracking method, significant interactions between view and any other factor suggested that the effects of the latter were moderated by the tracking method. For example, a significant view $\times$ treatment interaction would suggest that the effects of a given compound may vary as a function of how the behavior of the animal was scored (i.e., using 2D projections from top or front, or resorting to $3 \mathrm{D}$ trajectories). Upon detecting a significant interaction, we performed post-hoc comparisons, correcting for type-I errors, to detail whether and which pairwise comparisons were significant. Among these comparisons, those contrasting 2D and 3D were germane to the key question of the study.

This statistical model allowed testing the hypothesis that 2D views yielded spurious results compared to 3D data: false positive or false negative findings. Specifically, if 2D analysis reported a significant inter-group differences which was not confirmed by 3D data, we recorded such a result as a false positive. Likewise, if 2D analysis failed to identify significant inter-group differences, which were instead detected using the 3D approach, we recorded such a result as a

Peer] reviewing PDF | (2019:07:39852:1:0:NEW 28 Aug 2019) 
306 false negative. For example, consider the case in which we identified a significant time bins $\times$

307 view interaction where animals decreased their average speed as a function of time from 3D data

308 while maintaining their initial average speed throughout the trial from both the $2 \mathrm{D}$ views. In this

309 case, had post-hoc comparisons between 3D data and any of the 2D data confirmed ANOVA

310 results, we would register a false negative prediction for each of the $2 \mathrm{D}$ data. Instead, a false

311 positive finding would be registered in the opposite situation where 3D data indicated the lack of

312 a habituation profile in the average speed, while 2D data would support the opposite.

313

314

\section{Results}

315

316

317

318

319

320

321

322

323

324

325

326

327

328

329

330

331

332

333

334

\subsection{Ethanol and citalopram alter individual habituation to the test, measured through PCA on} $3 D$ trajectories

When analyzing the three components identified by PCA, scored from 3D trajectories, we observed that absolute levels of locomotion were indistinguishable between control and citalopram-treated subjects (condition: $F_{3,55}=0.52, P=0.668$ ) (Fig. 2a). Additionally, general locomotion steadily declined throughout the experimental session in all subjects (time: $F_{5,275}=$ $3.03, P=0.011 ; t_{275}>3.12, P<0.009$ ), regardless of the specific experimental group (time bins $\times$ condition: $\left.F_{15,275}=1.13, P=0.329\right)$. Absolute values of behavioral anxiety did not significantly vary across citalopram conditions (condition: $F_{3,55}=0.76, P=0.524$ ) (Fig. 2 b). Yet, it significantly decreased over the trial (time: $F_{5,275}=3.52, P=0.004 ; t_{275}>2.69, P<0.033$ ), albeit at a different rate (time bins $\times$ condition: $F_{15,275}=1.85, P=0.029$ ). Specifically, while behavioral anxiety remained constant throughout the experimental session in citalopram $50 \mathrm{mg} / \mathrm{L}$ and $100 \mathrm{mg} / \mathrm{L}$ conditions, it significantly declined in control and citalopram $30 \mathrm{mg} / \mathrm{L}$ conditions $\left(t_{275}>2.60, P<0.043\right)$. While positional anxiety did not significantly vary across citalopram conditions (condition: $F_{3,55}=0.95, P=0.421$ ) (Fig. 2c), it significantly increased over time (time: $F_{5,275}=3.51, P=0.004 ; t_{275}>2.82, P<0.023$ ). Such time-dependent profile varied depending on the experimental treatment (time bins $\times$ condition: $F_{15,275}=1.79, P=0.036$ ). Thus, while it remained constant in control and citalopram $100 \mathrm{mg} / \mathrm{L}$, it was low at the beginning of the test session and steadily increased in citalopram $30 \mathrm{mg} / \mathrm{L}$ and citalopram $50 \mathrm{mg} / \mathrm{L}$ subjects $\left(t_{275}>\right.$ 2.74, $P<0.029)$. 

statistically significant variation across experimental groups (condition: $F_{3,52}=2.43, P=0.072$ )

337 (Fig. 3a). When analyzing the time course of general locomotion, we observed that it significantly decreased over time (time: $F_{5,260}=3.02, P=0.011 ; t_{260}>2.67, P<0.035$ ), and that such a decrease was indistinguishable across all experimental groups (time bins $\times$ condition: $\left.F_{15,260}=1.50, P=0.105\right)$. Behavioral anxiety did not significantly vary across ethanol conditions (condition: $F_{3,52}=0.80, P=0.500$ ) (Fig. 3b), neither did it apparently change over time (time: $\left.F_{5,260}=1.66, P=0.144\right)$. However, we observed that the habituation profile varied depending on the specific experimental group (time bins $\times$ condition: $F_{15,260}=1.83, P=0.031$ ). Specifically, while behavioral anxiety remained constant in most experimental groups, it significantly declined over time in the ethanol $0.5 \%$ condition $\left(P<0.050 ; t_{260}=2.89 ; P=0.019\right)$. Finally, positional anxiety failed to reach a statistically significant variation across ethanol conditions (condition: $F_{3,52}=2.49, P=0.071$ ) (Fig. 3c), although it significantly decreased over time (time: $\left.F_{5,260}=3.25, P=0.007 ; t_{260}>2.75 ; P<0.029\right)$. Specifically, it significantly decreased for the ethanol $1.0 \%$ condition (time bins $\times$ condition: $F_{15,260}=2.33, P=0.004 ; t_{260}>3.76 ; P<0.001$ ).

\subsection{The scoring view influences the validity of experimental outcomes}

352

353

354

355

356

357

358

359

360

361

362 363

364

Herein, we report data concerning the effects of the views on all the experimental variables measured in the study. For the sake of clarity, in this section, we only report statistical findings associated with the scoring view (3D, 2D top, and 2D front) and its interactions with time or condition. Results concerning the main effects of condition, time, and their interaction irrespective of view are available in the supplementary material.

Before delving into detailed comparisons between the three different views for all the considered behavioral measures, we present an aggregated assessment of potentially inaccurate conclusions that would be drawn from 2D projections against 3D trajectories. The rate of false negative (erroneous reporting of absence of differences in lieu of significant findings in 3D) and false positive (erroneous reporting of significant differences in lieu of non-significantly different findings in 3D) findings is synoptically reported in Tables 2 and 3.

\subsubsection{Citalopram}


365 Average speed: Predictably, average speed varied significantly depending on which view was 366 used to compute it (view: $F_{2,110}=118.46, P<0.001$ ) (Fig. 4a). Specifically, both 2D front and 367 top views underestimated absolute levels of locomotion compared to $3 \mathrm{D}$ data $\left(t_{291.7}=9.87, P<\right.$ 3680.001 ; and $t_{291.7}=6.88, P<0.001$, respectively); additionally, $2 \mathrm{D}$ front view resulted in reduced 369 average speed compared to top view $\left(t_{291.7}=2.98, P=0.009\right)$. Experimental subjects did not 370 show a habituation profile to the test, yet 2D top projections indicated that the average speed 371 decreased from the first to the last minute (time bins $\times$ view: $F_{10,550}=14.08, P<0.001 ; t_{317.5}=$ $3723.03, P=0.012)$.

373 Average peak speed: Average peak speed was significantly underestimated in both 2D front and 374 top views in comparison with $3 \mathrm{D}$ data (view: $F_{2,110}=81.93, P<0.001 ; t_{346.6}>6.64, P<0.001$ )

375 (Fig. 4b). While the average peak speed decreased over time in all subjects (supplementary 376 material), experimental groups showed a differential habituation profile (time bins $\times$ view: $F_{10,550}$ $\left.377=15.64, P<0.001 ; t_{286.4}>2.73, P<0.029\right)$.

378 Average angular speed: Average angular speed was underestimated in the 2D front view 379 compared to both 3D and 2D top views (view: $F_{2,110}=88.45, P=0.001 ; t_{295.3}>5.33, P<0.001$ ) 380 (Fig. 4c). Furthermore, a decrease in average angular speed over time was observed in all views 381 (time bins $\times$ view: $F_{10,550}=7.40, P<0.001 ; t_{275}>2.69, P<0.034$ ).

382 383 384 385 386 387 388 389 390 391 392

Average peak angular speed: Average peak angular speed was underestimated when scored from 2D top view (view: $\left.F_{2,110}=10.09, P<0.001 ; t_{358.6}=3.62, P=0.001\right)$ (Fig. $4 d$ ). A decrease in average peak angular speed over time was recorded from all views, but not at the same times (time bins $\times$ view: $F_{2,550}=2.72, P=0.003 ; t_{275}>2.64, P<0.038$ ).

Average acceleration: Average acceleration was underestimated in both front and top 2D views compared to 3D (view: $F_{2,110}=126.62, P<0.001 ; t_{284.4}=9.24, P<0.001$; and $t_{284.4}=5.57, P<$ 0.001, respectively); additionally, 2D front view underestimated average acceleration compared to $2 \mathrm{D}$ top view $\left(t_{284.4}=3.67, P<0.001\right)$. Average acceleration varied over time depending on the view adopted to score fish behavior (time bins $\times$ view: $F_{10,550}=13.40, P<0.001$ ) (Fig. $4 \mathrm{e}$ ). Specifically, although average acceleration steadily declined from the third minute in ground truth $3 \mathrm{D}$ data $\left(t_{371.0}=2.57, P<0.046\right)$, such a decline was observable also from $2 \mathrm{D}$ top view 
$393\left(t_{371.0}>2.80, P<0.025\right)$, but only during the last minute in $2 \mathrm{D}$ front view $\left(t_{275.0}=2.70, P<\right.$ 394 0.033).

395 Average peak acceleration: Average peak acceleration significantly decreased over time,

396 regardless of the specific view adopted to compute this measure (time bins $\times$ view: $F_{10,550}=4.42$, $397 P<0.001 ; t_{344.7}>3.15, P<0.008$ ) (Fig. 4f). Yet, average peak acceleration was underestimated 398 in $2 \mathrm{D}$ front and top views compared to $3 \mathrm{D}$ (view: $F_{2,110}=79.67, P<0.001 ; t_{402.3}=6.05, P<$ 3990.001 ; and $t_{402.3}=3.46, P=0.002$, respectively). Additionally, 2D front view yielded a lower 400 average peak acceleration compared to top view $\left(t_{402.3}=2.59, P=0.027\right)$.

401 Wall following: Time spent wall following was significantly underestimated in both 2D front 402 view compared to 3D data (view: $F_{2,110}=237.90, P<0.001 ; t_{234.4}=17.55 ; P<0.001$ ).

403 Additionally, this metric was lower in $2 \mathrm{D}$ front view compared to $2 \mathrm{D}$ top view $\left(t_{234.4}=15.95, P\right.$ $404<0.001$ ) (Fig. 4g). While 3D and 2D top view data indicated that wall following increased 405 between the first and fifth minute of the experimental session (time bins $\times$ view: $F_{10,550}=2.16, P$ $\left.406=0.019, t_{824.7}>2.56, P<0.05\right), 2 \mathrm{D}$ front view data failed to identify this time dependent pattern 407 of thigmotaxis.

408 Position in the water column (proportion of time spent in the top half): Since this metric takes 409 into account only the vertical position of the fish, it cannot be scored from 2D top view and there 410 is no difference between values from 2D front view and 3D reconstructed trajectories (Fig. 4h 411 and 5h).

412 Freezing: Although the time spent freezing seemed to vary depending on which view was used to 413 compute it (view: $F_{2,110}=4.19, P=0.018$ ) (Fig. 4i), post-hoc tests revealed no pairwise

414 difference. Similarly, although an interaction between view and time was registered (time bins $\times$ 415 view: $F_{10,550}=2.35, P=0.010$ ), post-hoc comparisons did not indicate any specific difference.

\subsubsection{Ethanol}

418 Average speed: The different scoring views resulted in variable average speed values (view:

$\left.419 F_{2,104}=90.45, P<0.001\right)($ Fig. 5a). Both 2D top and front views underestimated average speed 420 compared to $3 \mathrm{D}\left(t_{174.3}=3.79 ; P<0.001\right.$; and $t_{174.3}=9.47 ; P<0.001$, respectively $)$.

421 Additionally, average speed was lower in 2D front view compared to top view $\left(t_{174.3}=5.69 ; P<\right.$ 
422 0.001). While data inspection suggested that habituation profiles were skewed by the view 423 adopted to score individual behavior (time bins $\times$ view: $F_{10,520}=10.14, P<0.001$ ), post-hoc 424 analyses failed to show significant view-dependent variations in this parameter.

425 Average peak speed: Average peak speed varied in all subjects, and this profile was apparently 426 influenced by the view adopted to score individual trajectories (time bins $\times$ view: $F_{10,520}=9.36$, $\left.427 P<0.001 ; t_{260.0}>2.64, P<0.039\right)$. This variation was manifested as a robust decline in subjects 428 treated with ethanol $0.50 \%$ concentration (time bins $\times$ condition $\times$ view: $F_{30,520}=1.83, P=$ $4290.005 ; t_{260.0}>2.57, P<0.047$, see Fig. S2, supplementary information). Furthermore, average 430 peak speed was significantly underestimated in both 2D front and top views compared to 3D data 431 (view: $F_{2,104}=83.2, P<0.001 ; t_{270.2}>4.41 ; P<0.001$ ) (Fig. 5b), as well as from the front view 432 compared to the top view $\left(t_{260.0}=2.60 ; P=0.027\right)$. Although ANOVA reported a significant 433 interaction between view and condition (condition $\times$ view: $F_{6,104}=2.33, P=0.037$ ), post-hoc 434 tests failed to reveal any significant pairwise difference.

435 Average angular speed: Predictably, both 2D front and top views underestimated average angular 436 speed compared to the $3 \mathrm{D}$ view (view: $F_{2,104}=63.33, P<0.001 ; t_{265.7}=7.73, P<0.001$; and $437 t_{265.7}=4.78, P<0.001$, respectively). Additionally, average angular speed was smaller in 2D 438 front view compared to $2 \mathrm{D}$ top view $\left(t_{265.7}=2.95, P<0.010\right)$ (Fig. 5c). Furthermore, 3D data 439 demonstrated that average angular speed declined between the first and the last minute of 440 observation. Such a decline, observable in 2D top view data, was not detected in 2D front view 441 (time bins $\times$ view: $F_{10,520}=5.19, P<0.001, t_{730.9}=3.902, P<0.005$ ).

442 Average peak angular speed: Although average peak angular speed appeared significantly 443 different depending on which view was used (view: $F_{2,104}=4.37, P=0.015$ ), such a difference 444 failed to emerge in post-hoc comparisons. From all views, average peak angular speed declined 445 throughout the experimental session (time bins $\times$ view: $F_{10,520}=3.53, P<0.001 ; t_{707.1}>2.66, P$ $446<0.035)$ (Fig. 5d).

447 Average acceleration: In line with most of the locomotion-related variables, average acceleration 448 was underestimated in both $2 \mathrm{D}$ front and top views compared to $3 \mathrm{D}$ view (view: $F_{2,104}=64.41, P$ $449<0.001 ; t_{164.6}=7.45, P<0.001$; and $t_{164.6}=3.20, P=0.005$, respectively, see Fig. 5e).

450 Furthermore, 2D front view yielded lower values of the average acceleration compared to the top 
451 view $\left(t_{164.6}=4.25, P<0.001\right)$. Individual habituation profile was differentially expressed by 452 experimental subjects depending on the specific view (time bins $\times$ view: $F_{10,520}=11.87, P<$ 453 0.001). Specifically, while 3D and 2D top view data indicated a general decrease in average 454 acceleration throughout the experimental session $\left(t_{312.7}=2.94, P<0.016\right)$, such a profile was not 455 visible in 2D front view, showing only a reduction during the third minute of the test $\left(t_{260.0}=\right.$ $4562.70, P=0.033)$. Data analysis suggested that the habituation profile varied depending on both 457 the view and the ethanol treatment (time bins $\times$ condition $\times$ view: $F_{30,520}=1.50, P=0.046$ ). 458 Specifically, we observed that the reduction in average acceleration was significant in ethanol $4590.5 \%\left(t_{260.0}>2.80, P<0.025\right)$, and that this decrease occurred regardless of the specific view 460 from which data were scored.

461 Average peak acceleration: Average peak acceleration varied depending on the specific scoring 462 view (view: $F_{2,104}=61.85, P<0.001$ ); specifically it was underestimated in both $2 \mathrm{D}$ front and 463 top views compared to $3 \mathrm{D}\left(t_{273.9}=5.69, P<0.001\right.$; and $t_{273.9}=2.41, P=0.044$, respectively $)$ and 464 was also less in 2D front view compared to $2 \mathrm{D}$ top view $\left(t_{273.9}=3.28, P=0.003\right)$ (Fig. 5f). 465 Furthermore, although data inspection suggested that the time-dependent habituation profile 466 varied depending on the specific view (time bins $\times$ view: $F_{10,520}=5.70, P<0.001 ; t_{312.6}=2.57$, $467 P<0.047)$, post-hoc tests did not support this suggestion. Thus, acceleration decreased with time 468 in experimental subjects regardless of the specific view adopted.

469 Wall following: The time spent in the proximity of the walls significantly varied depending on 470 the specific view used to compute it (view: $F_{2,104}=56.09, P<0.001$ ). Wall following was 471 significantly underestimated in both 2D front and top views compared to 3D $\left(t_{178.8}=9.96 ; P<\right.$ 4720.001 ; and $t_{178.8}=3.49 ; P=0.002$, respectively), and this parameter was less in $2 \mathrm{D}$ front view 473 compared to $2 \mathrm{D}$ top $\left(t_{178.8}=6.47, P<0.001\right)$. The individual habituation profiles varied 474 depending on the view (time bins $\times$ view: $F_{10,520}=3.37, P=0.001$ ) (Fig. 5g). Specifically, wall 475 following remained constant in 3D and 2D top view, and decreased in 2D front view $\left(t_{260.0}=\right.$ $4762.79 ; P<0.025)$. While wall following was apparently differed between conditions depending on 477 the scoring view (condition $\times$ view: $F_{2,104}=5.54, P<0.001$ ), such difference was not statistically 478 significant in pairwise comparisons. 
479 Freezing: While the time spent freezing seemed to vary depending on the specific scoring view, 480 (view: $F_{2,104}=5.35, P=0.006$ ) (Fig. 5i), such a difference was not confirmed by post-hoc tests 481 performed between the first and the sixth minute.

482

\section{Discussion}

The methodological nature of the present study first reverberated in the systematic evaluation of the correlation among the variables that constitute the ethogram exhibited in the novel tank diving test. The PCA revealed the presence of three orthogonal factors, reflecting general locomotion (average speed, average peak speed, average acceleration, and average peak acceleration), anxiety-related behavioral patterns (average angular speed, average angular peak speed, and freezing), and anxiety-related spatial preference (time spent close to the side walls and time spent in the upper half of the water column). The first principal component relates to the translational motion within the water tank. The behavioral patterns loading on the second principal component have been consistently associated with anxiety, in the form of erratic movements (zig-zagging) and freezing (Kalueff et al. 2013). From the catalog of Kalueff and colleagues (Kalueff et al. 2013), anxiety-related behavior is also related to thigmotaxis and geotaxis, which are the two behavioral measures that load on the third principal component.

While this analysis aligns with previous evidence indicating that anxiety can be expressed through different modalities, it also points at potential pitfalls of common practice in the construction of the ethogram of the novel tank diving test from 2D views. Specifically, the fact that variables contributing to the same principal component require different perspectives further corroborates the need for a 3D approach. For example, while position in the water column requires a front camera, wall distance and erratic movements need an overhead camera.

The analysis conducted on the aforementioned principal components revealed that both citalopram and ethanol influenced anxiety-related behaviors, thus corroborating the predictive validity of the novel tank diving test. Importantly, while citalopram concentration-dependently reduced locomotion and predictably reduced anxiety, ethanol resulted in increased anxiety, but only at a medium concentration (Tran et al. 2016c). Higher and lower ethanol concentrations were apparently ineffective. Low and medium concentrations of citalopram did not influence general locomotion but were associated with the exhibition of reduced anxiety, selectively during the first three minutes of testing. High concentrations of citalopram were associated with reduced 
510 locomotion and reduced anxiety throughout the entire test session. The anxiolytic effects of

511 citalopram have already been reported in several studies. For example, (Sackerman et al. 2010)

512 reported that zebrafish treated with $100 \mathrm{mg} / \mathrm{L}$ citalopram spent significantly more time than

513 control fish in the top two thirds of the tank, suggesting a decrease in anxiety compared to the

514 control.

515 It is worth noticing that, when analyzing discrete parameters rather than focusing on the

516 principal components, some anxiety-related behavioral parameters seemed unaffected by the

517 anxiolytic treatments applied. Specifically, we failed to observe a significant effect of citalopram

518 on the time spent in the upper portion of the tank, a classical measure of anxiety. We note that

519 such absence of a concentration-dependent behavioral response to anxiolytic compounds has also

520 been reported in other studies. For example, (Sackerman et al. 2010) reported that acute exposure

521 to $0.5 \%$ ethanol failed to alter the time spent in the upper portion of the test tank in zebrafish.

522 Similarly, (Maximino et al. 2011) failed to observe significant anxiety-related behavioral

523 alterations in response to fluoxetine. Finally, in a previous study, we also observed that $0.25 \%$

524 and $0.5 \%$ ethanol did not modulate anxiety-related behaviors in the light-dark test (Cianca et al.

525 2013). These false negative findings further corroborate the potential heuristic value of

526 conducting PCA in zebrafish behavioral pharmacology.

527 The anxiolytic effects of citalopram are likely related to its direct influence on

528 serotonergic concentrations. For example, handling stress has been shown to increase anxiety-

529 like behavior and reduce brain concentrations of the serotonin metabolite 5-HIAA (Tran et al.

530 2016b). Furthermore, (Maximino et al. 2014) observed that acute administration of the 5-HT1a

531 receptor agonist buspirone reduced behavioral anxiety in the light-dark test. Finally, in

532 accordance with the present study, the acute administration of the selective serotonin reuptake

533 inhibitor fluoxetine resulted in reduced anxiety in the geotaxis test (Maximino et al. 2013).

534 With respect to ethanol, available literature (Gerlai et al. 2000) indicates that its effects

535 vary depending on the concentration, administration schedule, and methodological issues.

536 (Pannia et al. 2014) documented the emergence of numerous ethanol-induced behavioral

537 changes, which are potentially influenced by zebrafish strain. Similar to our work, these changes

538 often manifest in the form of a complex habituation profile, where the behavior of the animal

539 varied as a function of time during the trial. (Tran et al. 2016a) reported that ethanol can have 
540 either anxiogenic or anxiolytic effects on zebrafish depending on whether the water in the test

541 tank comes from the individual's holding tank or from a tank that did not hold any fish.

542 Further, since ethanol influences general locomotion, some of its effects on anxiety may

543 be spurious and potentially related to locomotor effects. For example, a lack of vertical

544 exploration may reflect a decrease in swimming behavior due to the sedative effect of high

545 concentration of ethanol, rather than an anxiety response (Rosemberg et al. 2012). In our

546 previous study (Cianca et al. 2013), we observed that high ethanol concentration resulted in

547 reduced anxiety, associated with reduced motility and increased freezing. Likewise, (Gebauer et

548 al. 2011) observed that ethanol administration resulted in reduced anxiety in the light/dark test,

549 but not in the novel tank diving test. In contrast with these findings, Tran and collaborators (Tran

550 et al. 2016c) reported that acute exposure to high ethanol concentration resulted in increased

551 preference for the bottom of the test tank, and that such a variation related to alterations in brain

552 monoamines. Specifically, alcohol-treated subjects showed reduced concentrations of the

553 dopamine metabolite DOPAC, of serotonin and its metabolite 5-IAA (Tran et al. 2016c). Thus,

554 while the effects of ethanol on anxiety are more variable compared to those exerted by

555 citalopram, they apparently impinge on the same neurochemical pathways modulated by

556 citalopram. Ultimately, the complementary use of these substances served the aim to address the

557 validity of 2D approaches in zebrafish pharmacology of anxiety.

558 In order to compare 3D and 2D approaches, all experimental variables were also analyzed

559 independently from one-another. This comparison was aimed at confirming the intuition that

560 locomotion is underestimated when scoring the behavior in $2 \mathrm{D}$ and at assessing whether $2 \mathrm{D}$

561 views yielded incorrect conclusions regarding the effects of anxiolytics on individual behavior.

562 Working with raw experimental variables rather than aggregated principal components allowed

563 for a direct comparison of our findings with available literature, where the selected metrics are

564 routinely assessed in pharmacological phenotyping of zebrafish (Kalueff et al. 2013). With

565 respect to absolute values of locomotion, predictably, they were higher in $3 \mathrm{D}$ than $2 \mathrm{D}$, regardless

566 of whether the latter referred to the frontal or the horizontal plane. This can be easily explained

567 by recognizing that $2 \mathrm{D}$ trajectories correspond to the projection of the full $3 \mathrm{D}$ motion on

568 independent views, which would, by definition, abolish movement along a third dimension. This

569 evidence echoes our previous findings obtained in drug-free states (Macrì et al. 2017). 
The core objective of the present study was to evaluate whether $2 \mathrm{D}$ views may result in

571 inaccurate rejection of null hypotheses or acceptance of alternative ones. We observed that the

572 specific view consistently skewed the time course of the behavioral response to the novel tank.

573 This was reflected in the presence of ubiquitous significant view $\times$ time bins interactions across

574 most of the variables, and only few instances of view $\times$ condition interactions. Thus, these data

575 could preliminarily suggest a relative robustness of current scoring methods in zebrafish

576 pharmacology. Yet, in the light of the paucity of drug-dependent effects and of the nature of the

577 statistical model required to test the suitability of the 2D approaches compared to 3D, we argue

578 that this assessment only reflects a partial consideration of the observed results.

579 Specifically, in our previous study, we demonstrated that 2D experiments are

580 underpowered compared to $3 \mathrm{D}$ and therefore more prone to false negative findings than false

581 positive ones (Macrì et al. 2017). While in situations characterized by few significant main

582 effects of a given variable the likelihood to observe false negatives is intrinsically limited, data

583 with numerous significant main effects shall be amenable to the identification of numerous false

584 reporting instances. Accordingly, in the present study, the sporadic main effects of the condition

585 have apparently masked view-dependent false negatives; complementarily, the ubiquitous

586 presence of main effects of time bins allowed the detection of numerous view $\times$ time bins

587 interactions. Thus, the specific view from which data were scored influenced the observed

588 individual habituation patterns to the experimental paradigm. For example, while 3D data

589 indicated that locomotion-related parameters (e.g., speed, angular speed, and acceleration)

590 declined throughout the experimental session, 2D front view data failed to capture such a time-

591 dependent habituation pattern. While this aspect may simply indicate the limited heuristic

592 potential of the front view and advocate in favor of the use of a top view camera, we nonetheless

593 note that a front camera is indispensable to quantify the position in the water column, which

594 contributes to the anxiety-related phenotype.

595 These considerations extrapolate to zebrafish pharmacology, whereby our and others'

596 data (Cachat et al. 2010) indicate that anxiety-modulating compounds often alter habituation

597 profiles rather than absolute values averaged across different time points (Wong et al. 2010). For

598 example, we reported that anxiety-related behaviors in control subjects appear relatively constant

599 throughout the entire course of the experimental session. Conversely, experimental subjects

600 treated with low and medium concentrations of citalopram exhibit reduced anxiety-related 
601 behaviors during the early stages of the task, which gradually rise to attain control values

602 towards the end of the session. Similar to (Watts et al. 2017), we found that although 3D

603 measures offer higher precision, the benefit of using 3D compared to a view from the top is

604 limited regarding general behavioral pattern. The use of a front view remains necessary to

605 capture specific behaviors linked to the position of the fish in the water column.

606

607

608

\section{Conclusions}

In the present study, we examined whether the effort required to analyze zebrafish behavioral response to psychoactive compounds in 3D is warranted or a simpler 2D approach should be preferred. Such an effort resides in the use of two, rather than one, cameras and in the increased computational burden required when dealing with multiple views. The former aspect adds some limited costs to the experiment and requires some design considerations to ensure that the experimental tank is visually accessible from multiple locations. The latter aspect entails an increased workload by the experimenter to manually repair tracks that the behavioral scoring software had flagged during the automated analysis, as well as limited computational costs associated with synchronizing and fusing multiple videos.

Although not conclusive, our data suggest that 2D analysis might produce spurious predictions that might confound the generalizability of experimental results. We acknowledge that our claims are specific to an experimental tank which allowed fish to freely swim along the water column. Testing animals in shallow water, restricting behavioral patterns to $2 \mathrm{D}$, may not constitute an ideal solution, whereby it would add an unwanted source of distress to the animals that could mask the effect of the pharmacological manipulation. We also acknowledge that the experimental had a length threefold the width, which might have skewed the behavioral patterns of the animals with respect locomotion in the horizontal plane.

Finally, it is important to emphasize that in the present study we primarily focused on anxiolytic drugs and we thus cannot extrapolate our findings to the entire spectrum of anxietyrelated behaviors. Future studies are needed to test whether the considerations outlined in this eliciting stimuli (e.g., predators).

\section{Author contributions}


632 SM and MP designed the research; RJGC and CS performed the experiments; RJGC and CS

633 scored animal behavior; SM, RJGC, and MP performed statistical analyses; RJGC and CS

634 provided a preliminary draft; SM and MP wrote the final draft; and all the authors reviewed the

635 final draft and offered comments.

636

637 Acknowledgments

638 The authors are grateful to Hannah Kurdila for her help in data analysis, Shinnosuke Nakayama

639 for his help with the statistics, and Boris Arbuzov for his help in scoring fish behavior from the

640 video recordings.

641

642 Funding

643 This work was supported by the National Institutes of Health, National Institute on Drug Abuse

644 under grant number 1R21DA042558-01A1, the Office of Behavioral and Social Sciences

645 Research that co-founded the National Institute on Drug Abuse grant, and by the National

646 Science Foundation under grant number CMMI-1505832.

647

648 References

649 Abaid N, Bartolini T, Macri S, and Porfiri M. 2012. Zebrafish responds differentially to a robotic 650 fish of varying aspect ratio, tail beat frequency, noise, and color. Behavioural Brain $651 \quad$ Research 233:545-553. 10.1016/j.bbr.2012.05.047

652 Bateson M, Brilot B, and Nettle D. 2011. Anxiety: an evolutionary approach. Canadian Journal 653 of Psychiatry 56:707-715. 10.1177/070674371105601202

654

655

656

657

658

659

660

661

Bernier NJ, and Peter RE. 2001. The hypothalamic-pituitary-interrenal axis and the control of food intake in teleost fish. Comparative Biochemistry and Physiology Part B: Biochemistry and Molecular Biology 129:639-644.

Butail S, Bartolini T, and Porfiri M. 2013. Collective response of zebrafish shoals to a freeswimming robotic fish. PLoS One 8:e76123. 10.1371/journal.pone.0076123

Cachat J, Stewart A, Grossman L, Gaikwad S, Kadri F, Chung KM, Wu N, Wong K, Roy S, Suciu C, Goodspeed J, Elegante M, Bartels B, Elkhayat S, Tien D, Tan J, Denmark A, Gilder T, Kyzar E, Dileo J, Frank K, Chang K, Utterback E, Hart P, and Kalueff AV. 2010. 
662

663

664

665

666

667

668

669

670

671

672

673

674

675

676

677

678

679

680

681

682

683

684

685

686

687

688

689

690

691

692

Measuring behavioral and endocrine responses to novelty stress in adult zebrafish. Nature Protocol 5:1786-1799. 10.1038/nprot.2010.140

Cachat J, Stewart A, Utterback E, Hart P, Gaikwad S, Wong K, Kyzar E, Wu N, and Kalueff AV. 2011. Three-dimensional neurophenotyping of adult zebrafish behavior. PLoS One 6:e17597. 10.1371/journal.pone.0017597

Cahill GM. 1996. Circadian regulation of melatonin production in cultured zebrafish pineal and retina. Brain Research 708:177-181.

Caspi A, Sugden K, Moffitt TE, Taylor A, Craig IW, Harrington H, McClay J, Mill J, Martin J, Braithwaite A, and Poulton R. 2003. Influence of life stress on depression: moderation by a polymorphism in the 5-HTT gene. Science 301:386-389. 10.1126/science.1083968

Cianca V, Bartolini T, Porfiri M, and Macrì S. 2013. A robotics-based behavioral paradigm to measure anxiety-related responses in zebrafish. PLoS One 8:e69661. 10.1371/journal.pone.0069661

Davis M, Walker DL, Miles L, and Grillon C. 2010. Phasic vs sustained fear in rats and humans: role of the extended amygdala in fear vs anxiety. Neuropsychopharmacology 35:105-135. 10.1038/npp.2009.109

Delcourt J, Ovidio M, Denoël M, Muller M, Pendeville H, Deneubourg J-L, and Poncin P. 2018. Individual identification and marking techniques for zebrafish. Reviews in Fish Biology and Fisheries 18:839-864. 10.1007/s11160-018-9537-y

Egan RJ, Bergner CL, Hart PC, Cachat JM, Canavello PR, Elegante MF, Elkhayat SI, Bartels BK, Tien AK, Tien DH, Mohnot S, Beeson E, Glasgow E, Amri H, Zukowska Z, and Kalueff AV. 2009. Understanding behavioral and physiological phenotypes of stress and anxiety in zebrafish. Behavioural Brain Research 205:38-44. 10.1016/j.bbr.2009.06.022

Fontana BD, Mezzomo NJ, Kalueff AV, and Rosemberg DB. 2018. The developing utility of zebrafish models of neurological and neuropsychiatric disorders: A critical review. Experimental Neurology 299:157-171. 10.1016/j.expneurol.2017.10.004

Fossat P, Bacque-Cazenave J, De Deurwaerdere P, Delbecque JP, and Cattaert D. 2014. Comparative behavior. Anxiety-like behavior in crayfish is controlled by serotonin. Science 344:1293-1297. 10.1126/science.1248811

Franco-Restrepo JE, Forero DA, and Vargas RA. 2019. A Review of Freely Available, OpenSource Software for the Automated Analysis of the Behavior of Adult Zebrafish. Zebrafish. 
693 Gebauer DL, Pagnussat N, Piato AL, Schaefer IC, Bonan CD, and Lara DR. 2011. Effects of

694

695

696

697

698

699

700

701

702

703

704

705

706

707

708

709

710

711

712

713

714

715

716

717

718

719

720

721

722

723 anxiolytics in zebrafish: similarities and differences between benzodiazepines, buspirone and ethanol. Pharmacology Biochemistry and Behavior 99:480-486. 10.1016/j.pbb.2011.04.021

Gerlai R, Lahav M, Guo S, and Rosenthal A. 2000. Drinks like a fish: zebra fish (Danio rerio) as a behavior genetic model to study alcohol effects. Pharmacology Biochemistry and Behavior 67:773-782.

Haller J, and Alicki M. 2012. Current animal models of anxiety, anxiety disorders, and anxiolytic drugs. Current Opinion in Psychiatry 25:59-64. 10.1097/YCO.0b013e32834de34f

Hart PC, Bergner CL, Smolinsky AN, Dufour BD, Egan RJ, Laporte JL, and Kalueff AV. 2010. Experimental models of anxiety for drug discovery and brain research. Methods in Molecular Biology 602:299-321. 10.1007/978-1-60761-058-8_18

Hogg S, Paterson S, Mills AD, and File SE. 1994. Receptor binding in Japanese quail selected for long or short tonic immobility. Pharmacology Biochemistry and Behavior 49:625-628.

Howe K, Clark MD, Torroja CF, Torrance J, Berthelot C, Muffato M, Collins JE, Humphray S, McLaren K, Matthews L, McLaren S, Sealy I, Caccamo M, Churcher C, Scott C, Barrett JC, Koch R, Rauch GJ, White S, Chow W, Kilian B, Quintais LT, Guerra-Assuncao JA, Zhou Y, Gu Y, Yen J, Vogel JH, Eyre T, Redmond S, Banerjee R, Chi J, Fu B, Langley E, Maguire SF, Laird GK, Lloyd D, Kenyon E, Donaldson S, Sehra H, Almeida-King J, Loveland J, Trevanion S, Jones M, Quail M, Willey D, Hunt A, Burton J, Sims S, McLay K, Plumb B, Davis J, Clee C, Oliver K, Clark R, Riddle C, Elliot D, Threadgold G, Harden G, Ware D, Begum S, Mortimore B, Kerry G, Heath P, Phillimore B, Tracey A, Corby N, Dunn M, Johnson C, Wood J, Clark S, Pelan S, Griffiths G, Smith M, Glithero R, Howden P, Barker N, Lloyd C, Stevens C, Harley J, Holt K, Panagiotidis G, Lovell J, Beasley H, Henderson C, Gordon D, Auger K, Wright D, Collins J, Raisen C, Dyer L, Leung K, Robertson L, Ambridge K, Leongamornlert D, McGuire S, Gilderthorp R, Griffiths C, Manthravadi D, Nichol S, Barker G, Whitehead S, Kay M, Brown J, Murnane C, Gray E, Humphries M, Sycamore N, Barker D, Saunders D, Wallis J, Babbage A, Hammond S, Mashreghi-Mohammadi M, Barr L, Martin S, Wray P, Ellington A, Matthews N, Ellwood M, Woodmansey R, Clark G, Cooper J, Tromans A, Grafham D, Skuce C, Pandian R, Andrews R, Harrison E, Kimberley A, Garnett J, Fosker N, Hall R, Garner P, Kelly D, Bird 
724

725

726

727

728

729

730

731

732

733

734

735

736

737

738

739

740

741

742

743

744

745

746

747

748

749

750

751

752

753

754

C, Palmer S, Gehring I, Berger A, Dooley CM, Ersan-Urun Z, Eser C, Geiger H, Geisler M, Karotki L, Kirn A, Konantz J, Konantz M, Oberlander M, Rudolph-Geiger S, Teucke M, Lanz C, Raddatz G, Osoegawa K, Zhu B, Rapp A, Widaa S, Langford C, Yang F, Schuster SC, Carter NP, Harrow J, Ning Z, Herrero J, Searle SM, Enright A, Geisler R, Plasterk RH, Lee C, Westerfield M, de Jong PJ, Zon LI, Postlethwait JH, Nusslein-Volhard C, Hubbard TJ, Roest Crollius H, Rogers J, and Stemple DL. 2013. The zebrafish reference genome sequence and its relationship to the human genome. Nature 496:498-503. 10.1038/nature12111

Kalueff AV, Gebhardt M, Stewart AM, Cachat JM, Brimmer M, Chawla JS, Craddock C, Kyzar EJ, Roth A, Landsman S, Gaikwad S, Robinson K, Baatrup E, Tierney K, Shamchuk A, Norton W, Miller N, Nicolson T, Braubach O, Gilman CP, Pittman J, Rosemberg DB, Gerlai R, Echevarria D, Lamb E, Neuhauss SC, Weng W, Bally-Cuif L, Schneider H, and Zebrafish Neuroscience Research C. 2013. Towards a comprehensive catalog of zebrafish behavior 1.0 and beyond. Zebrafish 10:70-86. 10.1089/zeb.2012.0861

Kalueff AV, Stewart AM, and Gerlai R. 2014. Zebrafish as an emerging model for studying complex brain disorders. Trends in Pharmacological Science 35:63-75. 10.1016/j.tips.2013.12.002

Kalueff AV, Wheaton M, and Murphy DL. 2007. What's wrong with my mouse model? Advances and strategies in animal modeling of anxiety and depression. Behavioural Brain Research 179:1-18. 10.1016/j.bbr.2007.01.023

Kilkenny C, Browne WJ, Cuthill IC, Emerson M, and Altman DG. 2010. Improving bioscience research reporting: the ARRIVE guidelines for reporting animal research. PLoS Biology 8:e1000412. 10.1371/journal.pbio.1000412

Lee C, Verbeek E, Doyle R, and Bateson M. 2016. Attention bias to threat indicates anxiety differences in sheep. Biology Letters 12. 10.1098/rsbl.2015.0977

Lynn SE, and Kern MD. 2018. Interactions of body temperature and nutritional status on hypothalamo-pituitary-adrenal axis activity in pre-thermoregulatory eastern bluebird chicks (Sialia sialis). General and Comparative Endocrinology 267:82-89. 10.1016/j.ygcen.2018.06.003

Maaswinkel H, Zhu L, and Weng W. 2013. Using an automated 3D-tracking system to record individual and shoals of adult zebrafish. JoVE (Journal of Visualized Experiments):e50681. 
755

756

757

758

759

760

761

762

763

764

765

766

767

768

769

770

771

772

773

774

775

776

777

778

779

780

781

782

783

784

785

Macrì S, Neri D, Ruberto T, Mwaffo V, Butail S, and Porfiri M. 2017. Three-dimensional scoring of zebrafish behavior unveils biological phenomena hidden by two-dimensional analyses. Scientific Reports 7:1962. 10.1038/s41598-017-01990-Z

Macrì S, and Wurbel H. 2006. Developmental plasticity of HPA and fear responses in rats: a critical review of the maternal mediation hypothesis. Hormones and Behavior 50:667-680. 10.1016/j.yhbeh.2006.06.015

Maximino C, da Silva AWB, Araujo J, Lima MG, Miranda V, Puty B, Benzecry R, Picanco-Diniz DL, Gouveia A, Jr., Oliveira KR, and Herculano AM. 2014. Fingerprinting of psychoactive drugs in zebrafish anxiety-like behaviors. PLoS One 9:e103943. 10.1371/journal.pone.0103943

Maximino C, da Silva AWB, Gouveia A, Jr., and Herculano AM. 2011. Pharmacological analysis of zebrafish (Danio rerio) scototaxis. Progress in Neuro-Psychopharmacology and Biological Psychiatry 35:624-631.

Maximino C, Puty B, Benzecry R, Araujo J, Lima MG, de Jesus Oliveira Batista E, Renata de Matos Oliveira K, Crespo-Lopez ME, and Herculano AM. 2013. Role of serotonin in zebrafish (Danio rerio) anxiety: relationship with serotonin levels and effect of buspirone, WAY 100635, SB 224289, fluoxetine and para-chlorophenylalanine (pCPA) in two behavioral models. Neuropharmacology 71:83-97. 10.1016/j.neuropharm.2013.03.006

McCarroll MN, Gendelev L, Keiser MJ, and Kokel D. 2016. Leveraging large-scale behavioral profiling in zebrafish to explore neuroactive polypharmacology. ACS Chemical Biology 11:842-849. 10.1021/acschembio.5b00800

Mwaffo V, Butail S, di Bernardo M, and Porfiri M. 2015. Measuring zebrafish turning rate. Zebrafish 12:250-254. 10.1089/zeb.2015.1081

Nema S, Hasan W, Bhargava A, and Bhargava Y. 2016. A novel method for automated tracking and quantification of adult zebrafish behaviour during anxiety. Journal of Neuroscience Methods 271:65-75. 10.1016/j.jneumeth.2016.07.004

Nesse RM. 1999. Proximate and evolutionary studies of anxiety, stress and depression: synergy at the interface. Neuroscience \& Biobehavioral Reviews 23:895-903.

Pannia E, Tran S, Rampersad M, and Gerlai R. 2014. Acute ethanol exposure induces behavioural differences in two zebrafish (Danio rerio) strains: a time course analysis. Behavioural Brain Research 259:174-185. 10.1016/j.bbr.2013.11.006 
786 Parker KJ, Buckmaster CL, Lindley SE, Schatzberg AF, and Lyons DM. 2012. Hypothalamic-

787

788

789

790

791

792

793

794

795

796

797

798

799

800

801

802

803

804

805

806

807

808

809

810

811

812

813

814

815 pituitary-adrenal axis physiology and cognitive control of behavior in stress inoculated monkeys. International Journal of Behavioral Development 36. $10.1177 / 0165025411406864$

Perez-Escudero A, Vicente-Page J, Hinz RC, Arganda S, and de Polavieja GG. 2014. idTracker: tracking individuals in a group by automatic identification of unmarked animals. Nature Methods 11:743-748. 10.1038/nmeth.2994

Quinn GP, and Keough MJ. 2002. Experimental design and data analysis for biologists: Cambridge University Press.

Rodrigues SM, LeDoux JE, and Sapolsky RM. 2009. The influence of stress hormones on fear circuitry. Annual Review of Neuroscience 32:289-313. 10.1146/annurev.neuro.051508.135620

Rosemberg DB, Braga MM, Rico EP, Loss CM, Cordova SD, Mussulini BH, Blaser RE, Leite CE, Campos MM, Dias RD, Calcagnotto ME, de Oliveira DL, and Souza DO. 2012. Behavioral effects of taurine pretreatment in zebrafish acutely exposed to ethanol. Neuropharmacology 63:613-623. 10.1016/j.neuropharm.2012.05.009

Sackerman J, Donegan JJ, Cunningham CS, Nguyen NN, Lawless K, Long A, Benno RH, and Gould GG. 2010. Zebrafish behavior in novel environments: Effects of acute exposure to anxiolytic compounds and choice of Danio rerio line. Internal Journal of Comparative Psychology 23:43-61.

Shams S, Rihel J, Ortiz JG, and Gerlai R. 2018. The zebrafish as a promising tool for modeling human brain disorders: A review based upon an IBNS Symposium. Neuroscience \& Biobehavioral Reviews 85:176-190. 10.1016/j.neubiorev.2017.09.002

Spinello C, Macrì S, and Porfiri M. 2013. Acute ethanol administration affects zebrafish preference for a biologically inspired robot. Alcohol 47:391-398. 10.1016/j.alcohol.2013.04.003

Stewart AM, Braubach O, Spitsbergen J, Gerlai R, and Kalueff AV. 2014. Zebrafish models for translational neuroscience research: from tank to bedside. Trends in Neuroscience 37:264278. 10.1016/j.tins.2014.02.011

Stewart AM, Grieco F, Tegelenbosch RA, Kyzar EJ, Nguyen M, Kaluyeva A, Song C, Noldus LP, and Kalueff AV. 2015. A novel 3D method of locomotor analysis in adult zebrafish: 
816

817

818

819

820

821

822

823

824

825

826

827

828

829

830

831

832

833

834

835

836

837

838

839

840

Implications for automated detection of CNS drug-evoked phenotypes. Journal of Neuroscience Methods 255:66-74. 10.1016/j.jneumeth.2015.07.023

Tran S, Facciol A, and Gerlai R. 2016a. Home tank water versus novel water differentially affect alcohol-induced locomotor activity and anxiety related behaviours in zebrafish. Pharmacology Biochemistry and Behavior 144:13-19. 10.1016/j.pbb.2016.02.009

Tran S, and Gerlai R. 2013. Time-course of behavioural changes induced by ethanol in zebrafish (Danio rerio). Behavioural Brain Research 252:204-213. 10.1016/j.bbr.2013.05.065

Tran S, Nowicki M, Fulcher N, Chatterjee D, and Gerlai R. 2016b. Interaction between handling induced stress and anxiolytic effects of ethanol in zebrafish: A behavioral and neurochemical analysis. Behavioural Brain Research 298:278-285. 10.1016/j.bbr.2015.10.061

Tran S, Nowicki M, Muraleetharan A, Chatterjee D, and Gerlai R. 2016c. Neurochemical factors underlying individual differences in locomotor activity and anxiety-like behavioral responses in zebrafish. Progress in Neuro-Psychopharmacology \& Biological Psychiatry 65:25-33. 10.1016/j.pnpbp.2015.08.009

Watts I, Nagy M, Holbrook RI, Biro D, and Burt de Perera T. 2017. Validating two-dimensional leadership models on three-dimensionally structured fish schools. $R$ Soc Open Sci 4:160804. 10.1098/rsos.160804

Wong K, Elegante M, Bartels B, Elkhayat S, Tien D, Roy S, Goodspeed J, Suciu C, Tan J, Grimes C, Chung A, Rosenberg M, Gaikwad S, Denmark A, Jackson A, Kadri F, Chung KM, Stewart A, Gilder T, Beeson E, Zapolsky I, Wu N, Cachat J, and Kalueff AV. 2010. Analyzing habituation responses to novelty in zebrafish (Danio rerio). Behavioural Brain Research 208:450-457. 10.1016/j.bbr.2009.12.023 
841

842

843

844

845

846

847

848

849

850

851

852

853

854

855

856

857

858

859

860

861

862

863

864

865

866

867

868

869

870

871

\section{Figure Captions}

Figure 1. Trajectory for a single fish from a control trial. (A) top view, (B) 3D reconstructed trajectory obtained from synchronizing trajectories from top and front views, and (C) front view. The color of the trajectory denotes the evolution of the position of the fish along the six-minute trial. The axes dimensions are $29 \mathrm{~cm} \times 8.5 \mathrm{~cm} \times 13 \mathrm{~cm}$. Our an in-house developed tracking software (available for download at https://github.com/sach1tb/peregrine) was used in the analysis.

Figure 2. Principal components for the citalopram conditions. Mean \pm standard error for (A) locomotion, (B) behavioral anxiety, and (C) positional anxiety, over six-minute trials, showing overall variation, as well as for each concentration of citalopram (control $0 \mathrm{mg} / \mathrm{L}, 30$ $\mathrm{mg} / \mathrm{L}, 50 \mathrm{mg} / \mathrm{L}$, and $100 \mathrm{mg} / \mathrm{L}$ ) based on the reconstructed trajectories in 3D. Data were analyzed through a repeated measures ANOVA for split-plot designs. Filled symbols denote a significant difference $(P<0.05)$ from the first minute within each condition. Horizontal bar denotes a significant overall difference in time.

Figure 3. Principal components for the ethanol conditions. Mean \pm standard error for (A) locomotion, (B) behavioral anxiety, and (C) positional anxiety, over six-minute trials, showing overall variation, as well as for each concentration of ethanol (control $0 \%, 0.25 \%, 0.50 \%$, and $1.0 \%$ ) based on the reconstructed trajectories in 3D. Data were analyzed through a repeated measures ANOVA for split-plot designs. Filled symbols denote a significant difference $(P<$ 0.05) from the first minute within each condition. Horizontal bar denotes a significant overall difference in time.

Figure 4. Behavioral parameters for the citalopram conditions. Mean \pm standard error for (A) average speed, (B) average peak speed, (C) average angular speed, (D) average peak angular speed, (E) average acceleration, (F) average peak acceleration, $(G)$ proportion of time spent within $3 \mathrm{~cm}$ of walls, (H) proportion of time spent in the top half of the tank, and (I) proportion of time spent freezing, over six-minute trials aggregated for all citalopram conditions, computed from 2D front and top views, and 3D reconstructed trajectories. Data were analyzed through a 
872 repeated measures ANOVA for split-plot designs. Filled symbols denote a significant difference

$873(P<0.05)$ from the first minute within each condition. Horizontal bar denotes a significant

874 overall difference over time. Filled symbols in the top right corner of each panel indicate a

875 significant overall difference with respect to 3D data.

876

877 Figure 5. Behavioral parameters for the ethanol conditions. Mean \pm standard error for (A)

878 average speed, (B) average peak speed, (C) average angular speed, (D) average peak angular

879 speed, (E) average acceleration, (F) average peak acceleration, (G) proportion of time spent

880 within $3 \mathrm{~cm}$ of walls, $(\mathrm{H})$ proportion of time spent in the top half of the tank, and (I) proportion

881 of time spent freezing, over six-minute trials aggregated for all ethanol conditions, computed

882 from 2D front and top views, and 3D reconstructed trajectories. Data were analyzed through a

883 repeated measures ANOVA for split-plot designs. Filled symbols denote a significant difference

$884(P<0.05)$ from the first minute within each condition. Horizontal bar denotes a significant

885 overall difference over time. Filled symbols in the top right corner of each panel indicate a

886 significant overall difference with respect to 3D data.

887

888 


\section{Table Captions}

890

891 Table 1. Principal component analysis. Summary results from the principal component

892 analysis for citalopram and ethanol conditions. Principal components with eigenvalue larger than

8931 are shown. Loadings greater than 0.7 or smaller than -0.7 are emboldened; loadings smaller

894 than 0.1 in magnitude are not displayed.

895

896 Table 2. Number of false positive and false negative findings for citalopram. Number of

897 false positives and false negatives produced for each parameter when computed based on 2D top

898 view and front view data, for the citalopram conditions. A false positive indicates that the 2D

899 view (top or front) yields a significant result that is not supported by the 3D scoring approach. A

900 false negative indicates that the $2 \mathrm{D}$ view (top or front) fails to detect a significant result $(P<$

9010.05 ) that is instead evident from the 3D scoring approach.

902

903 Table 3. Number of false positive and false negative findings for ethanol. Number of false

904 positives and false negatives produced for each parameter when computed based on 2D top view

905 and front view data, for the citalopram conditions. A false positive indicates that the 2D view

906 (top or front) yields a significant result $(P<0.05)$ that is not supported by the 3D scoring

907 approach. A false negative indicates that the 2D view (top or front) fails to detect a significant

908 result that is instead evident from the 3D scoring approach.

909

910

911 
Figure 1

Trajectory for a single fish from a control trial.

(A) top view, (B) 3D reconstructed trajectory obtained from synchronizing trajectories from top and front views, and (C) front view. The color of the trajectory denotes the evolution of the position of the fish along the six-minute trial. The axes dimensions are $29 \mathrm{~cm} \times 8.5 \mathrm{~cm} \times$ $13 \mathrm{~cm}$. Our an in-house developed tracking software (available for download at https://github.com/sach1tb/peregrine ) was used in the analysis.

a

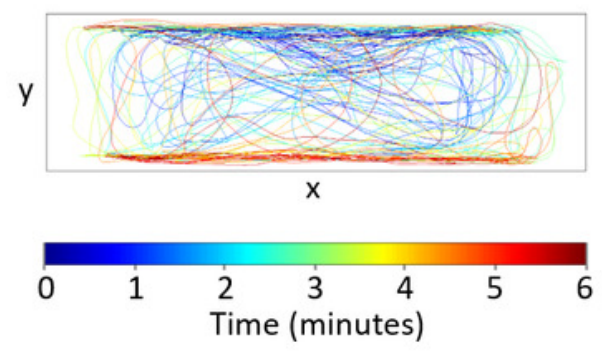

b

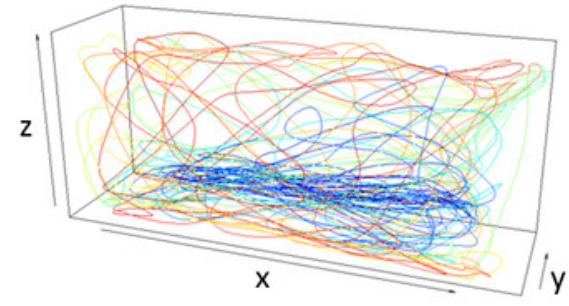

C

Z

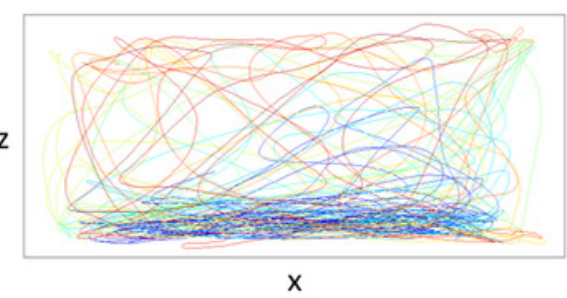




\section{Figure 2}

Principal components for the citalopram conditions.

Mean \pm standard error for (A) locomotion, (B) behavioral anxiety, and (C) positional anxiety, over six-minute trials, showing overall variation, as well as for each concentration of citalopram (control $0 \mathrm{mg} / \mathrm{L}, 30 \mathrm{mg} / \mathrm{L}, 50 \mathrm{mg} / \mathrm{L}$, and $100 \mathrm{mg} / \mathrm{L}$ ) based on the reconstructed trajectories in 3D. Data were analyzed through a repeated measures ANOVA for split-plot designs. Filled symbols denote a significant difference $(P<0.05)$ from the first minute within each condition. Horizontal bar denotes a significant overall difference in time
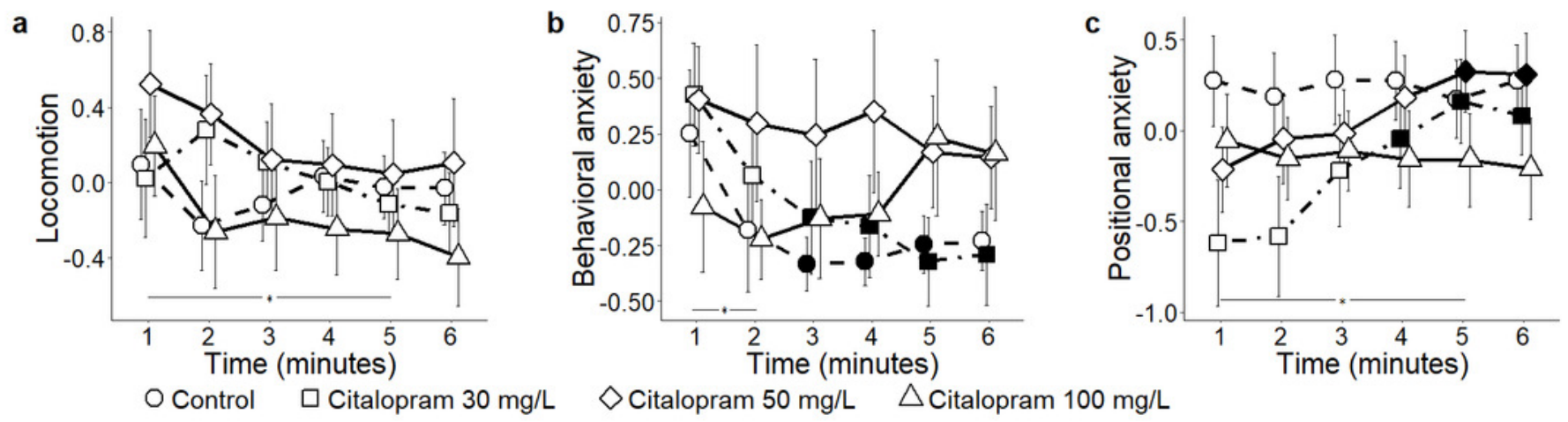

○ Control $\square$ Citalopram $30 \mathrm{mg} / \mathrm{L} \diamond$ Citalopram $50 \mathrm{mg} / \mathrm{L} \triangle$ Citalopram $100 \mathrm{mg} / \mathrm{L}$ 


\section{Figure 3}

Principal components for the ethanol conditions.

Mean \pm standard error for (A) locomotion, (B) behavioral anxiety, and (C) positional anxiety, over six-minute trials, showing overall variation, as well as for each concentration of ethanol (control $0 \%, 0.25 \%, 0.50 \%$, and 1.0\%) based on the reconstructed trajectories in 3D. Data were analyzed through a repeated measures ANOVA for split-plot designs. Filled symbols denote a significant difference $(P<0.05)$ from the first minute within each condition. Horizontal bar denotes a significant overall difference in time.
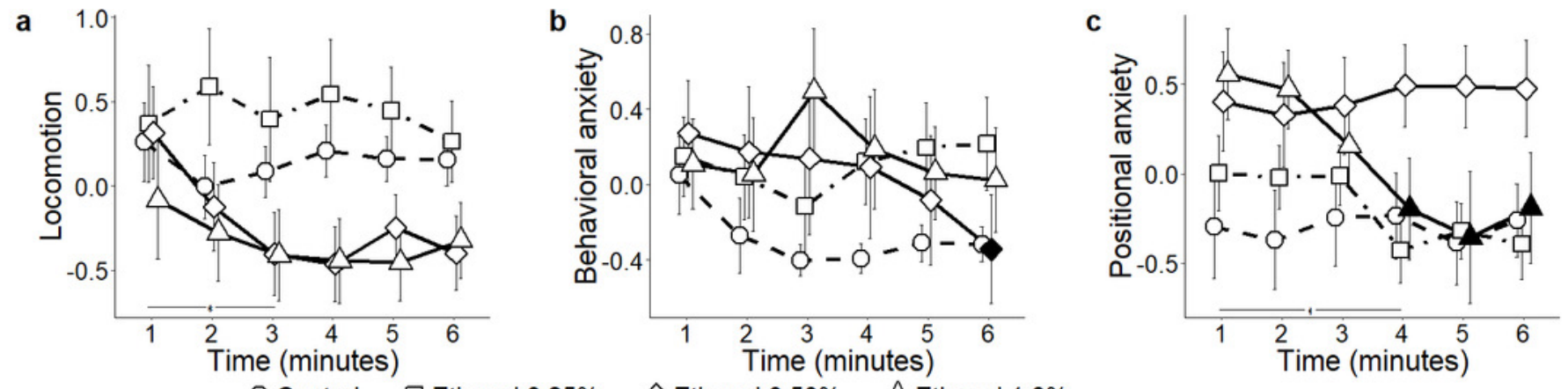

O Control $\square$ Ethanol $0.25 \% \triangle$ Ethanol $0.50 \% \triangle$ Ethanol $1.0 \%$ 


\section{Figure 4}

Behavioral parameters for the citalopram conditions.

Mean \pm standard error for (A) average speed, (B) average peak speed, (C) average angular speed, (D) average peak angular speed, (E) average acceleration, $(F)$ average peak acceleration, $(G)$ proportion of time spent within $3 \mathrm{~cm}$ of walls, $(H)$ proportion of time spent in the top half of the tank, and (I) proportion of time spent freezing, over six-minute trials aggregated for all citalopram conditions, computed from 2D front and top views, and 3D reconstructed trajectories. Data were analyzed through a repeated measures ANOVA for split-plot designs. Filled symbols denote a significant difference $(P<0.05)$ from the first minute within each condition. Horizontal bar denotes a significant overall difference over time. Filled symbols in the top right corner of each panel indicate a significant overall difference with respect to 3D data. 

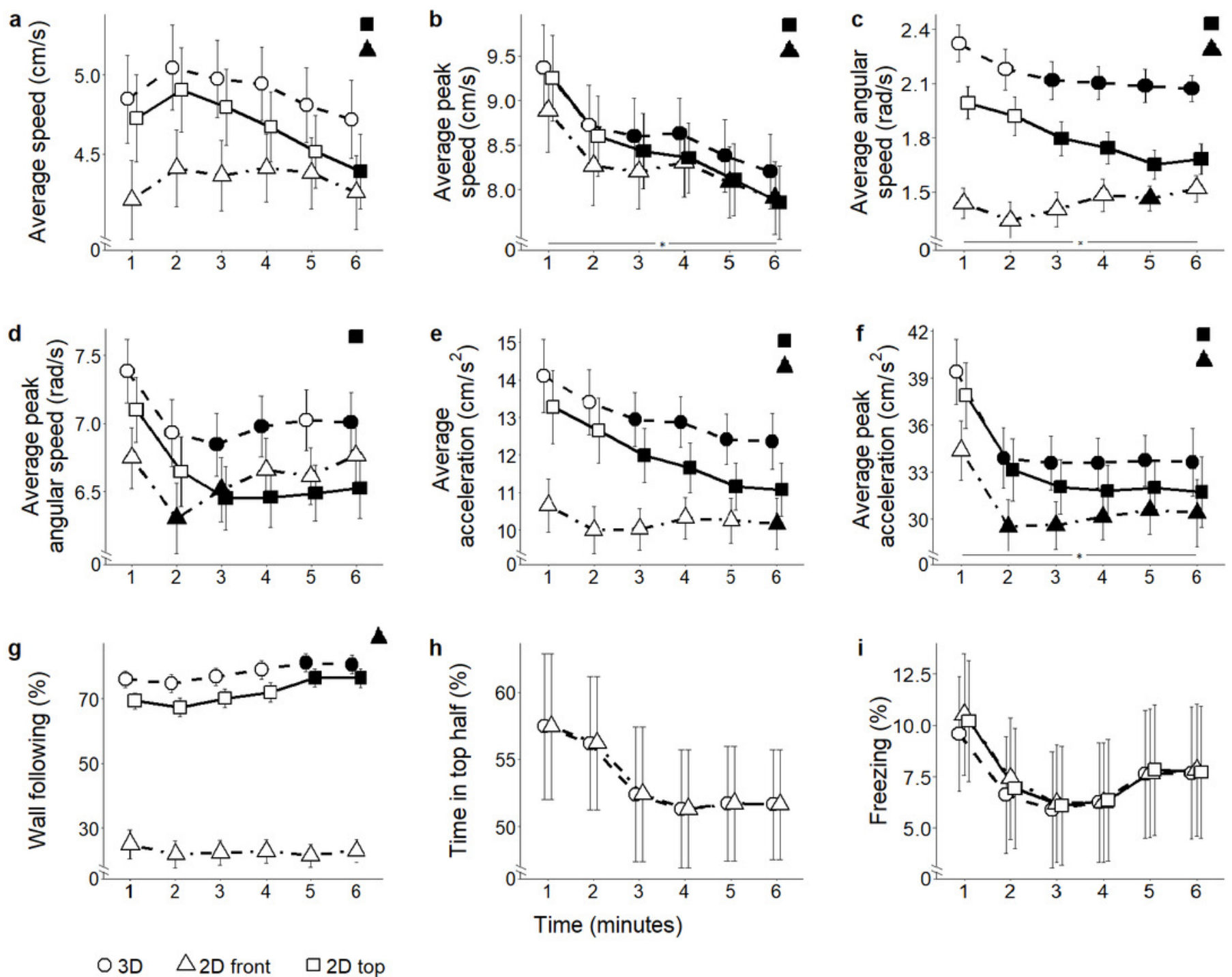

O 3D $\triangle 2 D$ front $\square 2 D$ top 


\section{Figure 5}

Behavioral parameters for the ethanol conditions.

Mean \pm standard error for (A) average speed, (B) average peak speed, (C) average angular speed, (D) average peak angular speed, (E) average acceleration, $(F)$ average peak acceleration, (G) proportion of time spent within $3 \mathrm{~cm}$ of walls, $(\mathrm{H})$ proportion of time spent in the top half of the tank, and (I) proportion of time spent freezing, over six-minute trials aggregated for all ethanol conditions, computed from 2D front and top views, and 3D reconstructed trajectories. Data were analyzed through a repeated measures ANOVA for split-plot designs. Filled symbols denote a significant difference $(P<0.05)$ from the first minute within each condition. Horizontal bar denotes a significant overall difference over time. Filled symbols in the top right corner of each panel indicate a significant overall difference with respect to 3D data. 

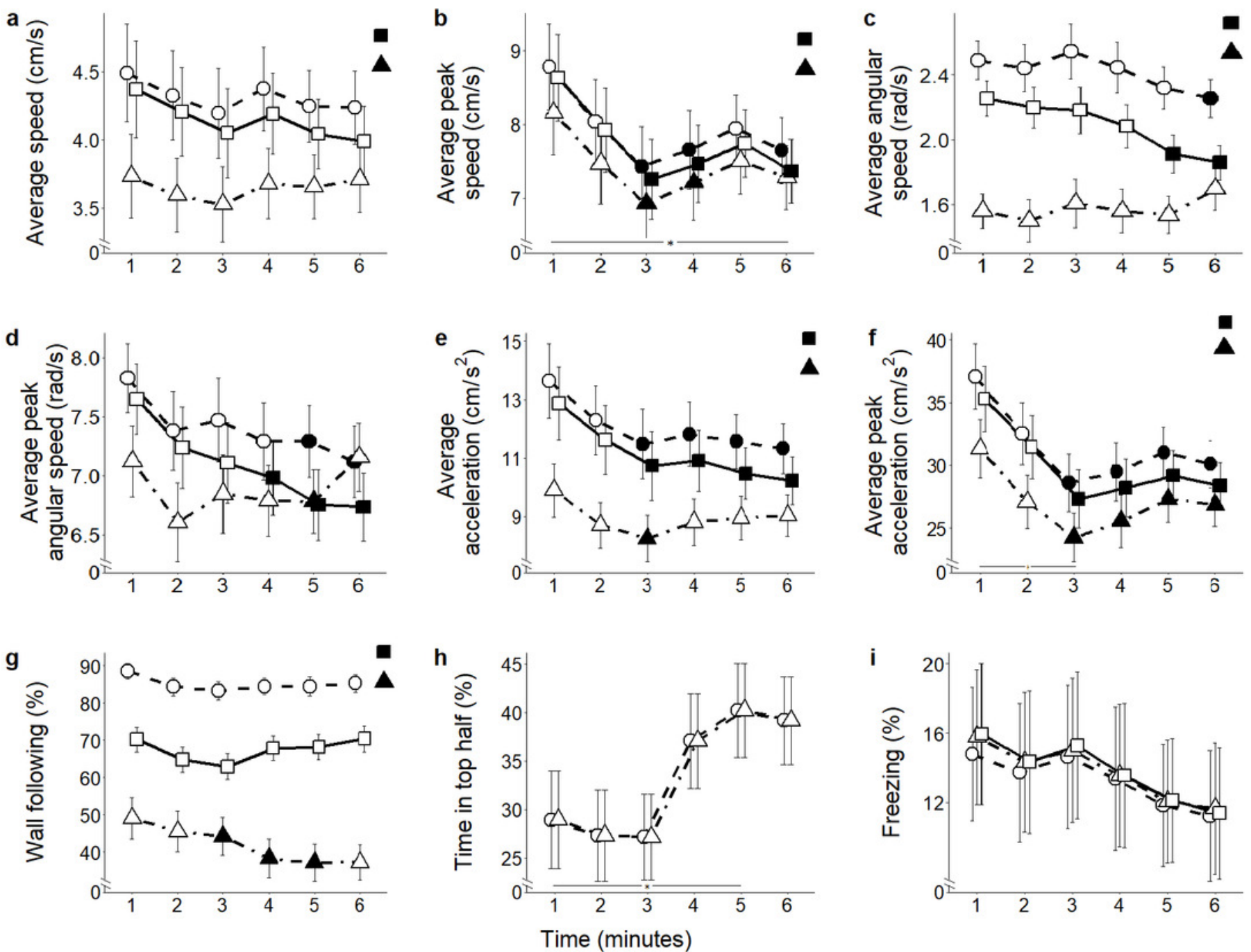

O 3D $\triangle 2 D$ front $\quad \square 2 D$ top 


\section{Table $\mathbf{1}$ (on next page)}

Principal component analysis.

Summary results from the principal component analysis for citalopram and ethanol conditions. Principal components with eigenvalue larger than 1 are shown. Loadings greater than 0.7 or smaller than -0.7 are emboldened; loadings smaller than 0.1 in magnitude are not displayed. 


\begin{tabular}{|c|c|c|c|c|c|c|}
\hline & \multicolumn{3}{|c|}{ Citalopram } & \multicolumn{3}{|c|}{ Ethanol } \\
\hline & Locomotion & $\begin{array}{c}\text { Behavioral } \\
\text { anxiety }\end{array}$ & $\begin{array}{l}\text { Positional } \\
\text { anxiety }\end{array}$ & Locomotion & $\begin{array}{l}\text { Behavioral } \\
\text { anxiety }\end{array}$ & $\begin{array}{l}\text { Positional } \\
\text { anxiety }\end{array}$ \\
\hline Eigenvalues & 4.29 & 2.34 & 1.19 & 4.29 & 2.47 & 1.06 \\
\hline $\begin{array}{l}\text { Explained variance } \\
(\%)\end{array}$ & 47.7 & 26.0 & 13.3 & 47.7 & 27.4 & 11.8 \\
\hline $\begin{array}{l}\text { Cumulative variance } \\
(\%)\end{array}$ & 47.7 & 73.6 & 86.9 & 47.7 & 75.1 & 86.9 \\
\hline \multicolumn{7}{|l|}{$\begin{array}{l}\text { Varimax-rotated } \\
\text { loadings }\end{array}$} \\
\hline Speed & 0.938 & -0.261 & & 0.924 & -0.289 & \\
\hline Average peak speed & 0.948 & -0.172 & & 0.953 & -0.122 & \\
\hline $\begin{array}{l}\text { Average angular } \\
\text { speed }\end{array}$ & & 0.939 & & & 0.946 & 0.106 \\
\hline $\begin{array}{l}\text { Average peak angular } \\
\text { speed }\end{array}$ & & 0.971 & & & 0.976 & 0.113 \\
\hline Average acceleration & 0.977 & & & 0.976 & & \\
\hline $\begin{array}{l}\text { Average peak } \\
\text { acceleration }\end{array}$ & 0.942 & & & 0.969 & & \\
\hline Freezing & -0.542 & 0.760 & & -0.524 & 0.746 & 0.170 \\
\hline Wall following & & 0.176 & 0.817 & & 0.309 & 0.670 \\
\hline Time in top half & -0.213 & & -0.788 & -0.118 & & -0.873 \\
\hline
\end{tabular}




\section{Table 2 (on next page)}

Number of false positive and false negative findings for citalopram.

Number of false positives and false negatives produced for each parameter when computed based on 2D top view and front view data, for the citalopram conditions. A false positive indicates that the 2D view (top or front) yields a significant result that is not supported by the $3 \mathrm{D}$ scoring approach. A false negative indicates that the 2D view (top or front) fails to detect a significant result that is instead evident from the 3D scoring approach. 


\begin{tabular}{l|ccc|ccc}
\hline Citalopram & \multicolumn{4}{l|}{$\begin{array}{l}\text { Differences between 3D and 2D top } \\
\text { view }\end{array}$} & \multicolumn{3}{l}{$\begin{array}{l}\text { Differences between 3D and 2D } \\
\text { front view }\end{array}$} \\
\hline Parameters & $\begin{array}{c}\text { False } \\
\text { positives }\end{array}$ & $\begin{array}{c}\text { False } \\
\text { negatives }\end{array}$ & Total & $\begin{array}{c}\text { False } \\
\text { positives }\end{array}$ & $\begin{array}{c}\text { False } \\
\text { negatives }\end{array}$ & Total \\
\hline Average speed & 1 & 0 & 1 & 0 & 0 & 0 \\
Average peak speed & 0 & 0 & 0 & 0 & 2 & 2 \\
Average angular speed & 0 & 0 & 0 & 0 & 3 & 3 \\
Average peak angular speed & 1 & 0 & 1 & 0 & 2 & 2 \\
Average acceleration & 0 & 0 & 0 & 0 & 3 & 3 \\
Average peak acceleration & 0 & 0 & 0 & 0 & 2 & 2 \\
Wall following & 0 & 0 & 0 & 0 & 2 & 2 \\
Time in top half & - & - & - & 0 & 0 & 0 \\
Freezing & 0 & 0 & 0 & 0 & 0 & 0 \\
\hline Total & 2 & 0 & 2 & 0 & 14 & 14 \\
\hline
\end{tabular}

1 


\section{Table 3 (on next page)}

Number of false positive and false negative findings for ethanol.

Number of false positives and false negatives produced for each parameter when computed based on 2D top view and front view data, for the citalopram conditions. A false positive indicates that the 2D view (top or front) yields a significant result that is not supported by the $3 \mathrm{D}$ scoring approach. A false negative indicates that the 2D view (top or front) fails to detect a significant result that is instead evident from the 3D scoring approach. 


\begin{tabular}{l|ccc|ccc}
\hline Ethanol & \multicolumn{4}{|l|}{$\begin{array}{l}\text { Differences between 3D and 2D top } \\
\text { view }\end{array}$} & \multicolumn{3}{l}{$\begin{array}{l}\text { Differences between 3D and 2D } \\
\text { front view }\end{array}$} \\
\hline Parameters & $\begin{array}{c}\text { False } \\
\text { positives }\end{array}$ & $\begin{array}{c}\text { False } \\
\text { negatives }\end{array}$ & Total & $\begin{array}{c}\text { False } \\
\text { positives }\end{array}$ & $\begin{array}{c}\text { False } \\
\text { negatives }\end{array}$ & Total \\
\hline Average speed & 0 & 0 & 0 & 0 & 0 & 0 \\
Average peak speed & 0 & 0 & 0 & 0 & 1 & 1 \\
Average angular speed & 1 & 0 & 1 & 0 & 1 & 1 \\
Average peak angular speed & 1 & 0 & 1 & 0 & 1 & 1 \\
Average acceleration & 0 & 0 & 0 & 3 & 0 & 3 \\
Average peak acceleration & 0 & 0 & 0 & 0 & 0 & 0 \\
Wall following & 0 & 0 & 0 & 3 & 0 & 3 \\
Time in top half & - & - & - & 0 & 0 & 0 \\
Freezing & 0 & 0 & 0 & 0 & 0 & 0 \\
\hline Total & 2 & 0 & 2 & 6 & 3 & 9 \\
\hline
\end{tabular}

1 